\title{
Recent Advances in Morphological Cell Image Analysis
}

\author{
Shengyong Chen, ${ }^{1}$ Mingzhu Zhao, ${ }^{2}$ Guang Wu, ${ }^{3,4}$ Chunyan Yao, ${ }^{1,5}$ and Jianwei Zhang ${ }^{5}$ \\ ${ }^{1}$ College of Computer Science and Technology, Zhejiang University of Technology, Hangzhou 310023, China \\ ${ }^{2}$ College of Information Engineering, Zhejiang University of Technology, Hangzhou 310023, China \\ ${ }^{3}$ Guangxi Academy of Sciences, 98 Daling Road, Nanning 530007, China \\ ${ }^{4}$ DreamSciTech Consulting, Shenzhen 518054, China \\ ${ }^{5}$ Department of Informatics, University of Hamburg, 22527 Hamburg, Germany
}

Correspondence should be addressed to Shengyong Chen, sy@ieee.org

Received 29 August 2011; Accepted 3 October 2011

Academic Editor: Carlo Cattani

Copyright ( 2012 Shengyong Chen et al. This is an open access article distributed under the Creative Commons Attribution License, which permits unrestricted use, distribution, and reproduction in any medium, provided the original work is properly cited.

This paper summarizes the recent advances in image processing methods for morphological cell analysis. The topic of morphological analysis has received much attention with the increasing demands in both bioinformatics and biomedical applications. Among many factors that affect the diagnosis of a disease, morphological cell analysis and statistics have made great contributions to results and effects for a doctor. Morphological cell analysis finds the cellar shape, cellar regularity, classification, statistics, diagnosis, and so forth. In the last 20 years, about 1000 publications have reported the use of morphological cell analysis in biomedical research. Relevant solutions encompass a rather wide application area, such as cell clumps segmentation, morphological characteristics extraction, $3 \mathrm{D}$ reconstruction, abnormal cells identification, and statistical analysis. These reports are summarized in this paper to enable easy referral to suitable methods for practical solutions. Representative contributions and future research trends are also addressed.

\section{Introduction}

Cell morphology has become a standard theory for computerized cell image processing and pattern recognition. The purpose of which is the quantitative characterization of cell morphology, including structure and inner-components analysis for better understanding functioning and pathogenesis associated with malignancy and behavior [1].

Morphological cell analysis is a key issue for abnormality identification and classification, early cancer detection, and dynamic changes analysis under specific environmental stress. The quantitative results and primary, objective, and reliable, which is beneficial to pathologists in making the final diagnosis and providing fast observation and automated analysis systems.

In the present study, advances in morphological cell analysis are briefly reviewed. Overall, significant progress has been made in several issues. Morphological cell analysis has been integrated in new methods for biomedical applications, such as automatic segmentation and analysis of histological tumour sections [2-4], boundary detection of cervical cell nuclei considering overlapping and clustering $[5,6]$, the granules segmentation and spatial distribution analysis [7], morphological characteristics analysis of specific biomedical cells $[8-10]$, understanding the chemotactic response and drug influences [11-14], or identifying cell morphogenesis in different cell cycle progression [15].

Morphological feature quantification for grading cancerous or precancerous cells is especially widely researched in the literature, such as nuclei segmentation based on marker-controlled watershed transform and snake model for hepatocellular carcinoma feature extraction and classification, which is important for prognosis and treatment planning [16], nuclei feature quantification for cancer cell cycle analysis [17], and using feature extraction including image morphological analysis, wavelet analysis, and texture analysis for automated classification of renal cell [18].

Computerized/automated early cancer or abnormalities detection provides a basis for reducing deaths and morbidity, especially for cervical cancer, which is reported to be 
the most preventable disease through early detection [19], provision of prompt advice, and opportunities for followup treatments. As an example, [20] presents a prototype expert system for automated segmentation and effective cervical cancer detection, providing primary, objective, and reliable diagnostic results to gynaecologists in making the final diagnosis. These advances will contribute to realize computer-assisted, interactive, or automated processing, quantification, statistic analysis, and diagnosis systems for biomedical applications.

The scope of this paper is restricted to morphological cell analysis by image processing in the field of biomedical research. Although this topic has attracted researchers as since early as the 1980s [21-23], this survey concentrates on the contributions of the last 5 years. No review of this nature can possibly cite each and every paper that has been published. Therefore, we include only what we believe to be representative samples of important works and broad trends from recent years. In many cases, references were provided to better summarize and draw distinctions among key ideas and approaches.

The paper has five more sections. Section 2 briefly provides an overview of related contributions. Section 3 introduces the typical formulation of cell morphology. Section 4 lists the relevant tasks, problems, and applications of cell morphology. Section 5 concentrates typical solutions and methods. Section 6 is a discussion of our impressions on current and future trends. Section 7 is the conclusion.

\section{Overview of Contributions}

2.1. Summary. From 1980s to 2010, about 1000 research papers with topics on or closely related to morphological cell analysis for robot vision were published. Figure 1 shows the yearly distribution of these published papers. The plot shows that the topic of morphological cell analysis steadily developed in the past 20 years.

2.2. Representatives. Morphological cell analysis has many applications in biomedical engineering. Their most significant roles are summarized as follows.

(1) Malignant cell identification and cancer detection $[20,24,25]$.

(2) Morphological changes during a cell cycle as division, proliferation, transition, and apoptosis [26-28] or to follow cell culture development [29].

(3) Morphological differences to elucidate the physiological mechanisms [30] or classify a set of cell populations with different functions such as neurons $[31,32]$.

(4) Dynamic characteristics investigation under specific environmental stress for personalized therapy [3336] or for the selection of new drugs [37].

(5) Morphometrical study such as subcellular structures (DNA, chromosome) analysis [38] for higher animals or plants based on 3D reconstruction $[39,40]$.

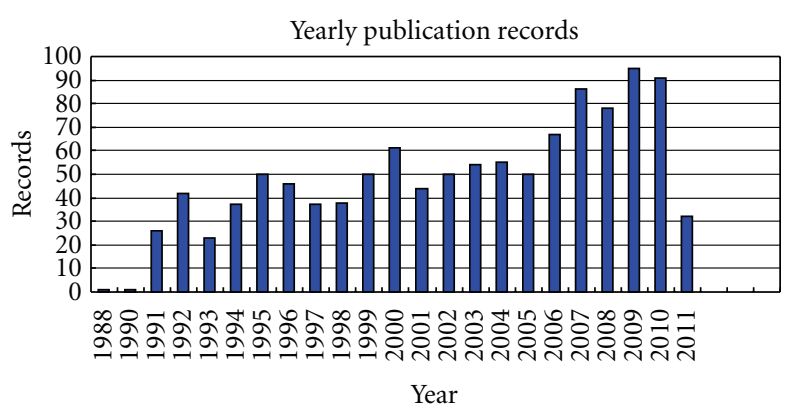

FIGURE 1: Yearly published records from 1990 to 2010.

TABLE 1: Representative contributions.

\begin{tabular}{llc}
\hline Processing & Method & Representative \\
\hline & $\begin{array}{l}\text { Active contour model (ACM) } \\
\text { Reconstruct the approximate } \\
\text { location of cellular } \\
\text { membranes }\end{array}$ & {$[5]-2011$} \\
Segmentation & $\begin{array}{l}\text { A marker-controlled } \\
\text { watershed transform and a } \\
\text { snake model } \\
\text { Segmentation combing } \\
\text { features }\end{array}$ & {$[16]-2010$} \\
\hline \multirow{3}{*}{ Classification } & $\begin{array}{l}\text { K-means and support vector } \\
\text { machines (SVM) } \\
\text { Bayesian classifier }\end{array}$ & {$[51]-2011$} \\
\hline
\end{tabular}

The commonly researched topics for solving morphological problems are listed below.

(1) Mathematical morphology theory used in binary, gray, and color images for preprocessing or features analysis [41-48].

(2) Location determination: objects located and analysis of distribution $[7,49,50]$.

(3) Meaningful areas segmentation: based on the features of pixel, edge, region, and model [2-4].

(4) Characteristics quantification: based on cytopathology and the experience of physicians [51-58].

(5) Recognition, classification automated analysis, and diagnosis $[6,16,24,51,59]$.

Morphological analysis has become a powerful mathematical tool for analyzing and solving cell informatics. Automatic features quantification is undoubtedly the most widely used estimation technique in this topic. Among the variety of developed methods, the main differences and remarkable features can be summarized briefly: shape, geometrical, intensity, and texture. A few representative types of segmentation and classification are selected for easy appreciation of state-of-the-art as shown in Table 1. 


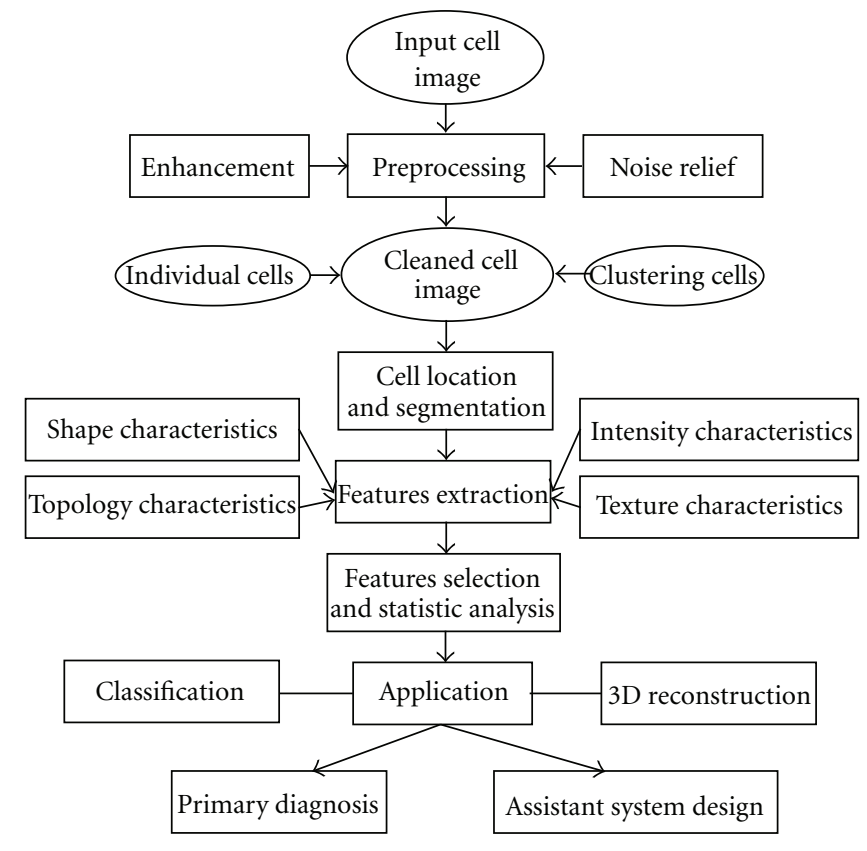

FIgURE 2: The general procedure of cell image analysis.

\section{The Problem and Fundamental Principle}

The fundamental principle of morphological cell analysis is dependent on cell biology, cytopathology, and the diagnostic experience of pathologists. To study cell characteristics, detect abnormalities, and determine the malignant degree, the pathologists examine biopsy material under a microscope, which is subjective, laborious, and time consuming. Therefore quantitative cell morphology is studied and computer-assisted systems are presented for diagnostic process at the same time. The general procedure of such applications can be described in Figure 2.

\section{Tasks and Problems}

4.1. Morphological Operation. Mathematical morphology is the basic theory for many image processing algorithms, which can also extract image shape features by operating with various shape-structuring elements [60]. This processing technique has proved to be a powerful tool for many computer-vision tasks in binary and gray scale images, such as edge detection, noise suppression, image enhancement, skeletonization, and pattern recognition, [45]. This technique is consisted of two parts: binary morphology and grayscale morphology, and the commonly used operations as morphological dilation and erosion are defined as follows, respectively:

$$
\begin{aligned}
& (f \oplus k)(x, y)=\max \{f(x-m, y-n)+k(m, n)\}, \\
& (f \Theta k)(x, y)=\max \{f(x-m, y-n)-k(m, n)\},
\end{aligned}
$$

where $f$ is the original image (gray scale or binary), which is operated by the corresponding structuring element $k$, and $(x, y)$ is the pixel of image $f,(m, n)$ is the size of element $k$. After morphological operation, image shape features such as edges, fillets, holes, corners, wedges, and cracks can be extracted.

Mathematical morphology can also be used in color images avoiding the loss of information of traditional binary techniques [45]. The new operations are based on the order in multivariate data processing.

4.2. Cell Localization. Determination of the orientation of a cell, termed localization, is of paramount importance in achieving reliable and robust morphological analysis. Achieving high-level tasks such as segmentation and shape description is possible if the initial position is known. From the early literature, primary methods were used in sample images, such as [61] using a sequence of morphological image operations to identify the cell nuclei and [29] using conditional dilation techniques to estimate unbiasedly cell density and obtain precisely cell contours. The results were acceptable only in single images without any complex factors.

Even when membranes are partially or completely not visible in the image (Figure 3(a)), the approximate locations of cells can be detected by reconstructing cellular membranes [51]. This method is effective for lung cells location in immunohistochemistry tissue images. Cell nuclei that are in cell clusters detecting are the key point for eliminating the positions of cervical cells in conventional Pap smear images (Figure 3(b)). To deal with this problem, Plissiti et al. present a fully automated method [6]. It takes the advantage of color information to obtain the candidate nuclei centroids in the images and eliminate the undesirable artifacts by applying a distance-dependent rule on the resulted centroids and classification algorithms (fuzzy C-means and support vector machines). The experiments shows that even in the case of 


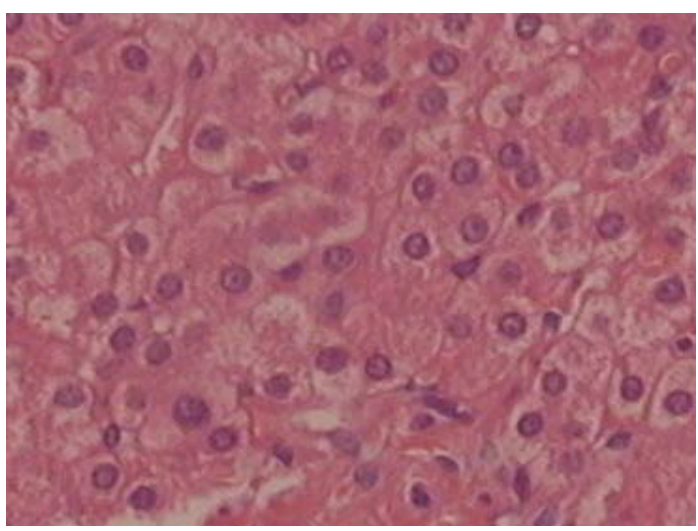

(a) Lung cells

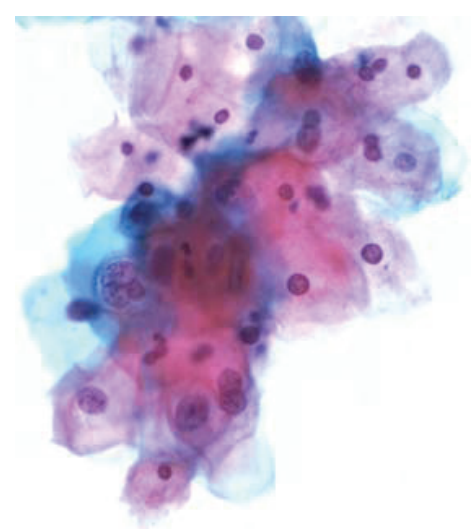

(b) Cervical cells

Figure 3: Biomedical cell images.

images with high degree of cell overlapping, the results are very promising.

For automatic detection of granules in different cell groups and statistical analysis of their spatial locations, the existing image analysis methods, such as single threshold, edge detection, and morphological operation, cannot be used. Thus, the empirical cumulative distribution function of the distances and the density of granules can be considered [7]. Jiang et al. propose a machine learning method [62], which is based on Haar feature (which is the combination of the intensity, shape, and scale information of the objects), to detect the particle's position.

4.3. Segmentation. Segmentation is one of the most important points for automated image analysis and better cell information understanding. The algorithms that have been presented can be divided into edge-based, region-based, and model-based modules. Region-based approaches attempt to segment an image into regions according to regional image data similarity (or dissimilarity), such as scale-space filtering, watershed clustering [63], gray-level threshold [26], and region growing [64]. For clear stained images, multilevel thresholds are the most simply and commonly applied methods for low-level segmentation to remove noise and obtain the interest region (nucleus, cytoplasm, or the whole cell), which are defined as follows:

$$
g(x, y)= \begin{cases}I_{i}, & T_{i-1} \leq f(m, n) \leq T_{i} \\ 0, & \text { others }\end{cases}
$$

where $i$ is the number of regions need to be divided, $T_{i}$ is the threshold and the extension ranges from $T_{i-1}$ to $T_{i}$ corresponding to the region $i$.

Nevertheless numerous algorithms have been developed, overlapping and connected cluster is still the key problem in cell image segmentation. The methods presented available to solve specific images with clear stained situation, semiautomated algorithms based on preknowledge for adequate segmentation of cell images under complex situation, are always more efficient than totally automated methods.
4.4. Quantitative Measurement of Meaningful Parameters. The quantitative measurement of cell features is meaningful for both image segmentation and abnormalities detection. Fast, reproducible, accurate, and objective measurement of cell morphology is beneficial to avoid subjective and interobserver variations, which result in diagnostic shifts and consequently disagreement between different interpreters [20]. The quantitative characteristics of cell or nuclear structure alterations extracted after robust image processing algorithms and $3 \mathrm{D}$ reconstruction is also called morphological biosignatures, which learn about cellular level features and nuclear structure including inner-components analysis, such as the quantitative evaluation of the approximate number of mRNA varying during cell cycle, developing, aging, and in different pathologies and treatment with drugs by extracting morphological parameters (cytoplasm and nucleus areas) [28]. Accurate quantification of these parameters could be beneficial for developing robust biosignatures for early cancer detection [1]. Multivariate statistical analyses of morphological data to suggest that quantitative cytology may be a useful adjunct to conventional tests for the selection of new drugs with differentiating potential [37].

The extracting features as cell area, perimeter, centroid, and the length of major and minor axes for calculating more meaningful parameters such as displacement, protrusiveness, and ellipticity, are used to analyze the dynamic changes of human cancerous glioma cells [35], which can also be used to identify different classed of neurons and relate neural structure (such as total dendritic length and dendritic field area) to function [31].

The most meaningful parameters are obtained in discriminating different patterns, such as cell size, shape distribution, and nuclear-to-cytoplasmic ratio for normal and precancerous cervical squamous epithelium determination [44], and texture quantification as a measurement to interchromosome coarseness to study cell proliferation [38]. Local gray level differences and cell density combining with other morphological parameters are possible to follow cell culture development under various experimental conditions [29]. Hitherto, the relationship between malignancyassociated morphological features in single tumour cells and 


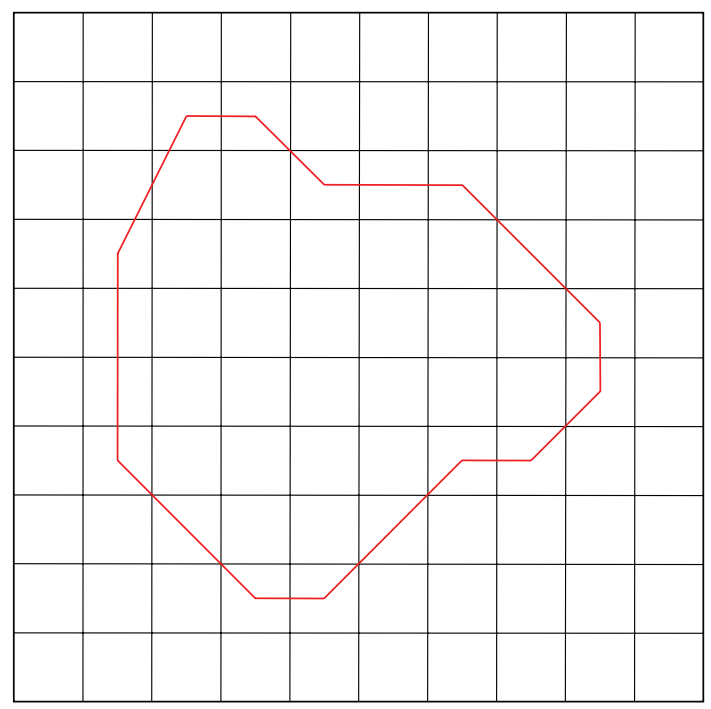

FIGURE 4: Geometrical features quantification.

the expression of markers indicating functional properties of these cells remained widely unknown [65].

4.5. Statistical Analysis. Multivariate statistic analysis is applied to compare multivariate data and establish the quantitative changes and differences between groups under investigation on their characteristics. The kernel approach is to find a high correlation feature set without redundancy. Principal components analysis (PCA) displays the original variables in a bidimensional space, thus reducing the dimensionality of the data and allowing the visualization of a large number of variables into a two-dimensional plot [11, 49, $66]$.

\section{Methods and Solutions}

5.1. Formulation in Morphological Analysis. Morphological analysis is often studied as the shape appearances of objects and the surfaces of the images, with intensity seen as height and texture appearing as relief. Formulization of morphological features is of benefit to computerized calculation and more efficient than manual morphological quantification, which is still laborious and subjective. The morphology characteristics can be described by shape, geometrical, intensity, and texture analysis.

The geometrical features of regions can be described by area, radii, perimeter, the major and the minor axis length, and so forth. The area of the object is calculated as the number of pixels of the region (Figure 4, the area defined by the closed curve). Radii are calculated based on projected cell area supposing that each cell is circular. The major and the minor axis length are the maximal and minimum numbers of pixels of the axis, respectively. Take Figure 4 as an example, the perimeter is calculated as follows:

$$
P=N_{1}+N_{2}+\sqrt{2} N_{3}
$$

where, $N_{1}, N_{2}, N_{3}$ are the numbers of the horizontal, vertical bevel lines on the boundary, respectively.

Circularity, rectangle, eccentricity, and irregularity are used to describe the shape features. Circularity $(C)$ and rectangle $(R)$ represent the rotundity-like and rectangle-like degree, defined as follows:

$$
\begin{gathered}
C=\frac{P^{2}}{4 \pi A}, \\
R=\frac{\text { Area }}{H * W} .
\end{gathered}
$$

Eccentricity is defined as follows:

$$
E=\frac{\text { The minor axis length }}{\text { The major axis length }} \text {. }
$$

Texture is an important visual cue and widely exists in images. Texture feature extraction is the most basic problem for texture analysis including classification and segmentation. Dimension, discrimination, stability, and calculation are considered in practical application and studied for more than fifty years. Based on the statistical theory, structure, model, and signal processing, many effective methods were presented for different applications. Among which, gray level co-occurrence matrix (GLCM) has become one of the best known and most widely used statistic method for texture feature extraction [26], especially in cell image texture feature analyzing. The interrelationship of textural primitives which define morphological texture can be estimated by quite different descriptors, the discriminant value of which varies considerably [67]. The descriptors based on GLCM are summarized in Table 2.

The intensity feature is characterized by the average of the intensity value of all the pixels of the region. For RGB color images, it is calculated independently from the red, green, and blue component of the original image. Histogram is an efficient way to show intensity features. Kruk et al. 
TABLE 2: Texture features.

\begin{tabular}{lc}
\hline Energy: & $\mathrm{ASM}=\sum_{i=1}^{k}\left(g_{i}-g\right)^{-2} p\left(g_{i}\right)$ \\
\hline Uniformity: & $U=\sum_{i=1}^{k} p^{2}\left(g_{i}\right)$ \\
\hline Entropy: & $\mathrm{ENT}=-\sum_{i=1}^{k} p\left(g_{i}\right) \log _{2} p\left(g_{i}\right)$ \\
\hline Smoothness:IDM $=1-1 /\left(1+s^{2}\right)$, where $s=\sqrt{\sum_{i=1}^{k}\left(g_{i}-g\right)^{-2} p\left(g_{i}\right)}$
\end{tabular}

Given that $g_{i}$ is the gray value, $k$ is the number of gray levels.

characterize the histograms of different color components by applying the following parameters: the mean, standard deviation, skewness, kurtosis, maximum value, and the span of the histogram [59].

5.2. Deformable Models. It is known that biomedical images are always under complex situation, which made segmentation a hard task for the extraction of the interest region. Because of the various challenges in medical image processing, deformable models were widely investigated and innovated, becoming a powerful tool for medical image segmentation. Active counter model is one of the most classical algorithms. Techniques based on active contour models have the potential to produce better estimates of cell morphologies.

The existing active contour models can be categorized into two classes: edge-based models [68], and region-based models [69]. On one hand edge-based model directly uses intensity gradient information to attract the contour toward the object boundaries. Therefore this kind of model has worse performance for weak object boundaries since cell image exhibits great fuzzy degree due to low contrast at the location of the cell membrane. On the other hand regionbased model aims to identify each region of interest by using a certain region descriptor. It guides the motion of the contour, and is less sensitive to the location of initial contours in some extents. It is much more suitable for cell segmentation than the fore one.

Chan and Vese model [70] is one of the most popular region-based active contour models. This model has been successfully used for segmenting images. Chan and Vese model proposed an active contour model that segments an image into two sets of possibly disjoint regions, by minimizing a simplified Mumford-Shah functional. The basic idea is as follows. Assume that $\Omega \subset R^{2}$ is the image domain and $I: \Omega \rightarrow \mathrm{R}$ is a given image. Mumford and Shah consider image segmentation as a problem of seeking an optimal contour $C$ that divides the image domain into two approximately piecewise-constant regions with intensities $u_{i}$ and $u_{0}$. Let $C$ denote its boundary. Thus the global data fitting term in the Chan and Vese model is defined as follows:

$$
E^{c v}\left(c_{1}, c_{2}\right)=\int_{\bar{\Omega}}\left(I-c_{1}\right)^{2} d x d y+\int_{\Omega}\left(I-c_{2}\right)^{2} d x d y,
$$

where $\Omega$ and $\bar{\Omega}$ represent the regions outside and inside the contour $C$, respectively, $c_{1}$ and $c_{2}$ are two constants that fit the image intensities outside $C$ and inside $C$.
This model considers pixels within the same region having the most similarity, and makes up the shortcomings of edged etector. When the contour accurately captures the object boundary, the two fitting terms minimize the fitting energy value. In each segmented area, the clustered pixels' mean value approximately equals the $c_{1}$ and $c_{2}$, respectively. Thus the fitting terms with respect to $c_{1}$ and $c_{2}$ are the driving forces that evolve the curve motion on the principle of innerregion homogeneity.

Since the regional difference is the guideline in image segmentation, the interregional differences should be considered as the model's driving force as follows:

$$
E=-\frac{1}{2}\left(c_{1}-c_{2}\right)^{2}
$$

This kind of region-based active contour model's energy is characterized by the maximum dissimilarity between regions. Minimizing the energy $E$ in (7) is the same as maximizing the difference between different regions. Equation (7) formulates the global instructive guidance term.

5.3. Classification. The extracted features involved the input to classification procedure for better analysis, correct grading, and pattern recognition. From the literature, unsupervised (as $K$-means and spectral clustering) and supervised (as super vector machine, SVM) classification schemes and artificial neural network (ANN) architecture were applied. SVM clustering is a state-of-the-art method, which was originally proposed in [71]. The decision function of a twoclass problem can be written as follows:

$$
f(x)=\omega \cdot \phi(x)+b=\sum_{i=1}^{N} \alpha_{i} y_{i} K\left(x, x_{i}\right)+b,
$$

where $x_{i} \in R^{d}$ is the sample and $y_{i} \in\{ \pm 1\}$ is the class label of $x_{i}$. A transformation $\phi(\cdot)$ maps the data points $x$ of the input space $R^{d}$ into a higher-dimensional feature space $R^{D}$, $(D \geq d) . K(\cdot, \cdot)$ is a kernel function, which defines an inner product in $R^{D} . K(\cdot, \cdot)$ is commonly defined as follows:

$$
\begin{gathered}
K\left(x, x_{i}\right)=\left[\left(x \cdot x_{i}\right)+1\right]^{q}, \\
K\left(x, x_{i}\right)=\exp \left\{-\frac{\left|x-x_{i}\right|^{2}}{\sigma^{2}}\right\}, \\
K\left(x, x_{i}\right)=\tanh \left(v\left(x \cdot x_{i}\right)+c\right) .
\end{gathered}
$$

The parameters $\alpha_{i} \geq 0$ are optimized by finding the hyperplane in feature space with maximum distance to the closest image $\phi\left(x_{i}\right)$ from the training set. For multilevel classification based on SVM, a decision-tree classification scheme discriminated between different grades is showed in Figure 5.

Although SVM is one of the most famous methods for classification and has achieved a great success in pattern recognition, problems still exist, such as the neglect of different data distributions within classes. Recently, structural super vector machine (SSVM) is proposed accordingly, which firstly exploits the intrinsic structures of samples 


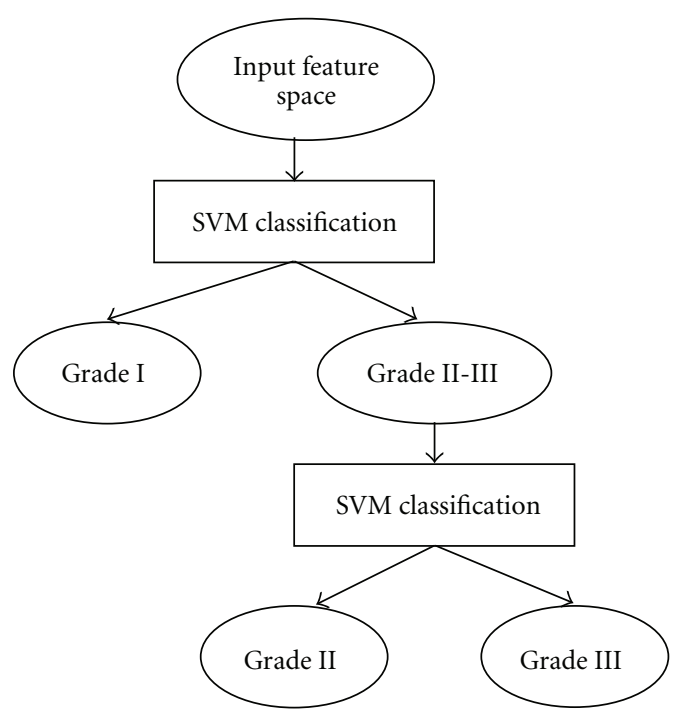

FIGURE 5: A decision-tree SVM classification scheme.

within classes by some unsupervised clustering methods and directly embedding the structural information into the SVM objective function [72]. SSVM is theoretically and empirically a better generalization than SVM algorithm.

5.4. D Morphology. Three-dimensional morphology using $3 \mathrm{D}$ reconstruction and image processing techniques is applied for quantitative morphometric analysis of cellular and subcellular structures, which is much more powerful than its 2D counterpart, but still largely based on the processing of separate $2 \mathrm{D}$ slices.

The approach to 3D morphological analysis consists of digital micrographs acquisition, reconstruction, and 3Dbased feature extraction. The acquired images are serialy taken by CT instrument at uniform angular intervals during a full $360^{\circ}$ rotation [1], from the electron imaging film taken by photo products [73], or by electron microscopy [40]. Computer programs such as MATLAB or Visual Studio software can be used for automated 3D image reconstruction.

Based on the reconstructed models, features such as three-dimensional shape of the cells can be extracted, which are correlated with the assembly state of myofibrils in different stages [74] and ultrastructure such as the arrangement of compact chromatin of GO lymphocytes can be studied [23].

\section{Existing Problems and Future Trends}

Although morphological cell analysis has been developed in many applications as mature approaches for estimation and diagnosis, some problems still exist in its applications in biomedical engineering. Researchers are exerting efforts not only in simple localization and segmentation, but also in improving the methods mainly in the following aspects.

\subsection{Real-Time Application and Computational Complexity.} Morphological cell analysis has been applied in almost all hospitals, which are key means in automatic microscopic analysis. However, because of its high computational complexity, it has strict limits on the number and stability of feature points. The traditional method selects a few features, which limits the application scope of morphological analysis. The computational complexity greatly affects realtime application systems $[50,75]$.

6.2. Reliability. Reliability is a great concern in practical applications [55, 76]. Morphological analysis relies on tuning of many parameters. Related techniques rely on existing noise statistics, initial positions, and sufficiently good approximation of measurement functions. Deviations from such assumptions usually lead to degraded estimations during automatic analysis. Stochastic stability is established in terms of the conditions of the initial errors, bound on observation noise covariance, observation nonlinearity, and modeling error. Features have to be effectively and efficiently treated by their removal from or addition to the system. New methods should be explored to discard outliers and improve the matching rate. These will help stabilize algorithms and allow more accurate localizations or parametric estimations.

6.3. With a Priori Knowledge. Constraints introduced in morphological cell parameters may help in some occasions. For example, morphological cell analysis is commonly used to estimate the cell shapes and activities, which incorporate a priori information in a consistent manner. However, the known model or information are often either ignored or heuristically dealt with [6].

6.4. Accuracy. Accuracy is always the most important factor in biomedical engineering. The accuracy of the calculated cells strongly depends on the computational potential and the statistical possibilities. For example, automated method provides accurate segmentation of the cellular membranes in the stained tracts and reconstructs the approximate location of the unstained tracts using nuclear membranes as a spatial 
reference. Accurate cell-by-cell membrane segmentation allows per-cell morphological analysis and quantification of the target membrane $[16,51,77]$.

6.5. Artificial Intelligence. The integration of the morphological cell analysis with some artificial intelligence methods may yield a better performance. Fuzzy logic, neural network, genetic algorithm, and so forth can be combined to wholly resolve the complex task.

\section{Conclusion}

This paper summarizes recent advances in morphological cell analysis for biomedical engineering applications. Typical contributions are addressed for initialization, localization, segmentation, estimation, modeling, shape analysis, cell parameters, and so forth. Representative works are listed for readers to have a general overview of state-of-the art. A number of methods for solving morphological problems are investigated. Many methods developed for morphological cell analysis, extended morphological cell segmentation, are introduced. In the 20-year history of morphological cell analysis, they gained entry into the field of biomedical engineering as a critical role. The largest volume of published reports in this literature belongs to the last ten years.

\section{Acknowledgments}

This paper was supported in part by the National Natural Science Foundation of China and Microsoft Research Asia (NSFC nos. 61173096, 51075367, and 60870002), Zhejiang Provincial S\&T Department (2010R10006, 2010C33095), and Zhejiang Provincial Natural Science Foundation of China (R1110679).

\section{References}

[1] V. Nandakumar, L. Kelbauskas, R. Johnson, and D. Meldrum, "Quantitative characterization of preneoplastic progression using single-cell computed tomography and threedimensional karyometry," Cytometry Part A, vol. 79, no. 1, pp. 25-34, 2011.

[2] C. C. Reyes-Aldasoro, L. J. Williams, S. Akerman, C. Kanthou, and G. M. Tozer, "An automatic algorithm for the segmentation and morphological analysis of microvessels in immunostained histological tumour sections," Journal of Microscopy, vol. 242, no. 3, pp. 262-278, 2011.

[3] J. Z. Cheng, Y. H. Chou, C. S. Huang et al., "ACCOMP: augmented cell competition algorithm for breast lesion demarcation in sonography," Medical Physics, vol. 37, no. 12, pp. 6240-6252, 2010.

[4] J. S. Schildkraut, N. Prosser, A. Savakis et al., "Level-set segmentation of pulmonary nodules in megavolt electronic portal images using a CT prior," Medical Physics, vol. 37, no. 11, pp. 5703-5710, 2010.

[5] M. E. Plissiti, C. Nikou, and A. Charchanti, "Combining shape, texture and intensity features for cell nuclei extraction in Pap smear images," Pattern Recognition Letters, vol. 32, no. 6, pp. 838-853, 2011.
[6] M. E. Plissiti, C. Nikou, and A. Charchanti, "Automated detection of cell nuclei in Pap smear images using morphological reconstruction and clustering," IEEE Transactions on Information Technology in Biomedicine, vol. 15, no. 2, pp. 233$241,2011$.

[7] E. Díaz, G. Ayala, M. E. Díaz, L. W. Gong, and D. Toomre, "Automatic detection of large dense-core vesicles in secretory cells and statistical analysis of their intracellular distribution," IEEE/ACM Transactions on Computational Biology and Bioinformatics, vol. 7, no. 1, Article ID 4468698, pp. 2-11, 2010.

[8] F. Brun, A. Accardo, M. Marchini, F. Ortolani, G. Turco, and S. Paoletti, "Texture analysis of TEM micrographs of alginate gels for cell microencapsulation," Microscopy Research and Technique, vol. 74, no. 1, pp. 58-66, 2011.

[9] S. Amini, D. Veilleux, and I. Villemure, "Tissue and cellular morphological changes in growth plate explants under compression," Journal of Biomechanics, vol. 43, no. 13, pp. 25822588, 2010.

[10] Y. Xiong, C. Kabacoff, J. Franca-Koh, P. N. Devreotes, D. N. Robinson, and P. A. Iglesias, "Automated characterization of cell shape changes during amoeboid motility by skeletonization," BMC Systems Biology, vol. 4, article 33, 2010.

[11] Y. A. Xiong and P. A. Iglesias, "Tools for analyzing cell shape changes during chemotaxis," Integrative Biology, vol. 2, no. 1112 , pp. 561-567, 2010.

[12] Z. Q. Hong, L. M. Tao, and L. Li, "Effect of stress on mRNA expression of H+-ATPase in osteoclasts," Molecular and Cellular Biochemistry, vol. 343, no. 1-2, pp. 183-190, 2010.

[13] Y. S. Lin, C. C. Lin, Y. S. Tsai, T. C. Ku, Y. H. Huang, and C. N. Hsu, "A spectral graph theoretic approach to quantification and calibration of collective morphological differences in cell images," Bioinformatics, vol. 26, no. 12, Article ID btq194, pp. i29-i37, 2010.

[14] P. Venkatesan, S. Das, M. M. R. Krishnan, C. Chakraborty, K. Chaudhury, and M. Mandal, "Effect of AEE788 and/or Celecoxib on colon cancer cell morphology using advanced microscopic techniques," Micron, vol. 41, no. 3, pp. 247-256, 2010.

[15] S. Ohnuki, S. Nogami, and Y. Ohya, "A microfluidic device to acquire high-magnification microphotographs of yeast cells," Cell Division, vol. 4, article 5, 2009.

[16] P. W. Huang and Y. H. Lai, "Effective segmentation and classification for HCC biopsy images," Pattern Recognition, vol. 43, no. 4, pp. 1550-1563, 2010.

[17] F. H. Li, X. B. Zhou, J. W. Ma, and S. T. C. Wong, "Multiple nuclei tracking using integer programming for quantitative cancer cell cycle analysis," IEEE Transactions on Medical Imaging, vol. 29, no. 1, Article ID 5175475, pp. 96-105, 2010.

[18] Q. Chaudry, S. H. Raza, A. N. Young, and M. D. Wang, "Automated renal cell carcinoma subtype classification using morphological, textural and wavelets based features," Journal of Signal Processing Systems, vol. 55, no. 1-3, pp. 15-23, 2009.

[19] M. Arbyn, M. Rebolj, I. M. C. M. De Kok et al., "The challenges of organising cervical screening programmes in the 15 old member states of the European Union," European Journal of Cancer, vol. 45, no. 15, pp. 2671-2678, 2009.

[20] S. W. K. Chan, K. S. Leung, and W. S. F. Wong, "An expert system for the detection of cervical cancer cells using knowledge-based image analyzer," Artificial Intelligence in Medicine, vol. 8, no. 1, pp. 67-90, 1996.

[21] L. Ficsor, V. S. Varga, A. Tagscherer, Z. Tulassay, and B. Molnar, "Automated classification of inflammation in colon histological sections based on digital microscopy and advanced image analysis," Cytometry Part A, vol. 73, no. 3, pp. 230-237, 2008. 
[22] A. Tárnok, G. K. Valet, and F. Emmrich, "Systems biology and clinical cytomics: the 10th Leipziger Workshop and the 3rd International Workshop on Slide-Based Cytometry," Cytometry Part A, vol. 69, no. 1, pp. 36-40, 2006.

[23] G. López-Velázquez, J. Márquez, E. Ubaldo, G. Corkidi, O. Echeverría, and G. H. Vázquez Nin, "Three-dimensional analysis of the arrangement of compact chromatin in the nucleus of $\mathrm{G}_{0}$ rat lymphocytes," Histochemistry and Cell Biology, vol. 105, no. 2, pp. 153-161, 1996.

[24] D. Glotsos, P. Spyridonos, D. Cavouras, P. Ravazoula, P. A. Dadioti, and G. Nikiforidis, "An image-analysis system based on support vector machines for automatic grade diagnosis of brain-tumour astrocytomas in clinical routine," Medical Informatics and the Internet in Medicine, vol. 30, no. 3, pp. 179193, 2005.

[25] C. O. De Solórzano, S. Costes, D. E. Callahan, B. Parvin, and M. H. Barcellos-Hoff, "Applications of quantitative digital image analysis to breast cancer research," Microscopy Research and Technique, vol. 59, no. 2, pp. 119-127, 2002.

[26] G. A. Losa and C. Castelli, "Nuclear patterns of human breast cancer cells during apoptosis: characterisation by fractal dimension and co-occurrence matrix statistics," Cell and Tissue Research, vol. 322, no. 2, pp. 257-267, 2005.

[27] T. Q. Xie, M. L. Zeidel, and Y. T. Pan, "Detection of tumorigenesis in urinary bladder with optical coherence tomography: optical characterization of morphological changes," Optics Express, vol. 10, no. 24, pp. 1431-1443, 2002.

[28] M. Colombi, L. Moro, N. Zoppi, and S. Barlati, "Quantitative evaluation of mRNAs by in situ hybridization and image analysis: principles and applications," DNA and Cell Biology, vol. 12, no. 7, pp. 629-636, 1993.

[29] W. Y. Xu-Van Opstal, C. Ranger, O. Lejeune et al., "Automated image analyzing system for the quantitative study of living cells in culture," Microscopy Research and Technique, vol. 28, no. 5, pp. 440-447, 1994.

[30] P. A. Melrose, C. Pickel, H. S. Cheramie, W. G. Henk, M. A. Littlefield-Chabaud, and D. D. French, "Distribution and morphology of immunoreactive gonadotropin-releasing hormone (GnRH) neurons in the basal forebrain of ponies," Journal of Comparative Neurology, vol. 339, no. 2, pp. 269-287, 1994.

[31] M. F. Villa and F. R. Amthor, "Automating the quantitative analysis of 2-D neural dendritic trees," Journal of Neuroscience Methods, vol. 56, no. 1, pp. 77-88, 1995.

[32] M. Masseroli, A. Bollea, and G. Forloni, "Quantitative morphology and shape classification of neurons by computerized image analysis," Computer Methods and Programs in Biomedicine, vol. 41, no. 2, pp. 89-99, 1993.

[33] M. C. Albertini, A. Accorsi, L. Teodori et al., "Use of multiparameter analysis for Vibrio alginolyticus viable but nonculturable state determination," Cytometry Part A, vol. 69, no. 4, pp. 260-265, 2006.

[34] E. Vlodavsky, E. Palzur, and J. F. Soustiel, "Hyperbaric oxygen therapy reduces neuroinflammation and expression of matrix metalloproteinase- 9 in the rat model of traumatic brain injury," Neuropathology and Applied Neurobiology, vol. 32, no. 1 , pp. 40-50, 2006.

[35] K. A. Giuliano, "Dissecting the individuality of cancer cells: the morphological and molecular dynamics of single human glioma cells," Cell Motility and the Cytoskeleton, vol. 35, no. 3, pp. 237-253, 1996.

[36] B. Rousset, Y. Munari-Silem, V. Gire, and P. Fonlupt, "Dynamic analysis of drug action on in vitro reconstituted thyroid follicle by microinjection of tracer molecules and videomicroscopy," Cell Biology and Toxicology, vol. 8, no. 3, pp. $1-7,1992$.

[37] C. Broglio, J. Dufer, P. Joly, Y. Carpentier, and A. Desplaces, "Quantitative morphological assessment of erythroblastic differentiation induced, in vitro, in human K562 leukemic cells," Analytical Cellular Pathology, vol. 5, no. 3, pp. 135-146, 1993.

[38] L. Vega-Alvarado, J. Márquez, and G. Corkidi, "Interchromosome texture as a feature for automatic identification of metaphase spreads," Medical and Biological Engineering and Computing, vol. 40, no. 4, pp. 479-484, 2002.

[39] N. Kutsuna and S. Hasezawa, "Morphometrical study of plant vacuolar dynamics in single cells using three-dimensional reconstruction from optical sections," Microscopy Research and Technique, vol. 68, no. 5, pp. 296-306, 2005.

[40] D. A. Winkelmann, T. S. Baker, and I. Rayment, "Threedimensional structure of myosin subfragment-1 from electron microscopy of sectioned crystals," Journal of Cell Biology, vol. 114, no. 4, pp. 701-713, 1991.

[41] T. G. Li, S. P. Wang, and N. Zhao, "Gray-scale edge detection for gastric tumor pathologic cell images by morphological analysis," Computers in Biology and Medicine, vol. 39, no. 11, pp. 947-952, 2009.

[42] J. Poikonen and A. Paasio, "An $8 \times 8$ cell analog order-statisticfilter array with asynchronous grayscale morphology in 0.13 $\mu \mathrm{m}$ CMOS," IEEE Transactions on Circuits and Systems I, vol. 56, no. 8, pp. 1541-1553, 2009.

[43] J. Angulo and S. Matou, "Application of mathematical morphology to the quantification of in vitro endothelial cell organization into tubular-like structures," Cellular and Molecular Biology, vol. 53, no. 2, pp. 22-35, 2007.

[44] D. C. Walker, B. H. Brown, A. D. Blacket, J. Tidy, and R. H. Smallwood, "A study of the morphological parameters of cervical squamous epithelium," Physiological Measurement, vol. 24, no. 1, pp. 121-135, 2003.

[45] F. Ortiz, F. Torres, E. De Juan, and N. Cuenca, "Colour mathematical morphology for neural image analysis," RealTime Imaging, vol. 8, no. 6, pp. 455-465, 2002.

[46] S. Li, K. H. Hu, N. Cai et al., "Automatic analysis of image of surface structure of cell wall-deficient EVC," Bio-Medical Materials and Engineering, vol. 11, no. 3, pp. 159-166, 2001.

[47] V. Metzler, T. Lehmann, H. Bienert, K. Mottaghy, and K. Spitzer, "Scale-independent shape analysis for quantitative cytology using mathematical morphology," Computers in Biology and Medicine, vol. 30, no. 3, pp. 135-151, 2000.

[48] G. Diaz, A. Zucca, M. D. Setzu, and C. Cappai, "Chromatin pattern by variogram analysis," Microscopy Research and Technique, vol. 39, no. 3, pp. 305-311, 1997.

[49] S. Holmes, A. Kapelner, and P. P. Lee, "An interactive java statistical image segmentation system: gemIdent," Journal of Statistical Software, vol. 30, no. 10, pp. 1-20, 2009.

[50] J. B. Hendricks, "Quantitative histology by laser scanning cytometry," Journal of Histotechnology, vol. 24, no. 1, pp. 5962, 2001.

[51] E. Ficarra, S. Di Cataldo, A. Acquaviva, and E. Macii, "Automated segmentation of cells with IHC membrane staining," IEEE Transactions on Biomedical Engineering, vol. 58, no. 5, pp. 1421-1429, 2011.

[52] K. S. Cheng, C. J. Chien, M. H. Hsu, and C. L. Li, "Development of a PC12 cell cultivation and monitoring system for neuronal-like study," Biomedical Engineering, vol. 22, no. 3, pp. 193-203, 2010.

[53] O. Schmitt and M. Hasse, "Morphological multiscale decomposition of connected regions with emphasis on cell clusters," 
Computer Vision and Image Understanding, vol. 113, no. 2, pp. 188-201, 2009.

[54] O. Schmitt and M. Hasse, "Radial symmetries based decomposition of cell clusters in binary and gray level images," Pattern Recognition, vol. 41, no. 6, pp. 1905-1923, 2008.

[55] C. López, M. Lejeune, M. T. Salvadó et al., "Automated quantification of nuclear immunohistochemical markers with different complexity," Histochemistry and Cell Biology, vol. 129, no. 3, pp. 379-387, 2008.

[56] P. Thurner, R. Müller, G. Raeber, U. Sennhauser, and J. A. Hubbell, "3D morphology of cell cultures: a quantitative approach using micrometer synchrotron light tomography," Microscopy Research and Technique, vol. 66, no. 6, pp. 289-298, 2005.

[57] G. Landini and I. E. Othman, "Architectural analysis of oral cancer, dysplastic, and normal epithelia," Cytometry Part A, vol. 61, no. 1, pp. 45-55, 2004.

[58] R. Nakajima, T. Nakamura, M. Ogawa, H. Miyakawa, and Y. Kudo, "Novel method for quantification of brain cell swelling in rat hippocampal slices," Journal of Neuroscience Research, vol. 76, no. 5, pp. 723-733, 2004.

[59] M. Kruk, S. Osowski, and R. Koktysz, "Recognition and classification of colon cells applying the ensemble of classifiers," Computers in Biology and Medicine, vol. 39, no. 2, pp. 156-165, 2009.

[60] F. Y. Shih, C. T. King, and C. C. Pu, "Pipeline architectures for recursive morphological operations," IEEE Transactions on Image Processing, vol. 4, no. 1, pp. 11-18, 1995.

[61] J. L. Humm, R. M. Macklis, Y. Yang, K. Bump, and L. M. Chin, "Image analysis for the study of radionuclide distribution in tissue sections," Journal of Nuclear Medicine, vol. 35, no. 7, pp. 1217-1225, 1994.

[62] S. Jiang, X. Zhou, T. Kirchhausen, and S. T. C. Wong, "Detection of molecular particles in live cells via machine learning," Cytometry Part A, vol. 71, no. 8, pp. 563-575, 2007.

[63] K. Jiang, Q. M. Liao, and Y. Xiong, "A novel white blood cell segmentation scheme based on feature space clustering," Soft Computing, vol. 10, no. 1, pp. 12-19, 2006.

[64] H. S. Wu, R. Xu, N. Harpaz, D. Burstein, and J. Gil, "Segmentation of intestinal gland images with iterative region growing," Journal of Microscopy, vol. 220, no. 3, pp. 190-204, 2005.

[65] G. Haroske, V. Dimmer, K. Friedrich et al., "Nuclear image analysis of immunohistochemically stained cells in breast carcinomas," Histochemistry and Cell Biology, vol. 105, no. 6, pp. 479-485, 1996.

[66] J. M. Higgins, D. T. Eddington, S. N. Bhatia, and L. Mahadevan, "Statistical dynamics of flowing red blood cells by morphological image processing," PLoS Computational Biology, vol. 5, no. 2, Article ID e1000288, 2009.

[67] M. Garcia-Bonafe and A. Moragas, "Differential diagnosis of malignant and reactive cells from serous effusions: image and texture analysis study," Analytical Cellular Pathology, vol. 12, no. 2, pp. 85-98, 1996.

[68] C. Li, C. Xu, C. Gui, and M. D. Fox, "Level set evolution without re-initialization: a new variational formulation," in Proceedings of the IEEE Computer Society Conference on Computer Vision and Pattern Recognition (CVPR '05), pp. 430436, June 2005.

[69] A. Tsai, A. Yezzi, and A. S. Willsky, "Curve evolution implementation of the Mumford-Shah functional for image segmentation, denoising, interpolation, and magnification," IEEE Transactions on Image Processing, vol. 10, no. 8, pp. 11691186, 2001.
[70] T. Chan and L. Vese, "Active contours without edges," IEEE Transactions on Image Processing, vol. 10, no. 2, pp. 266-277, 2001.

[71] Asa Ben-Hur, D. Horn, H. T. Siegelmann, and V. Vapnik, "Support vector machine clustering," Journal of Machine Learning Research, vol. 2, pp. 125-137, 2001.

[72] H. Xue, S. Chen, and Q. Yang, "Structural support vector machine," Lecture Notes in Computer Science, vol. 5263, no. 1, pp. 501-511, 2008.

[73] G. Zellnig, A. Perktold, and B. Zechmann, "Fine structural quantification of drought-stressed Picea abies (L.) organelles based on 3D reconstructions," Protoplasma, vol. 243, no. 1-4, pp. 129-136, 2010.

[74] J. M. Messerli, M. E. Eppenberger-Eberhardt, B. M. Rutishauser et al., "Remodelling of cardiomyocyte cytoarchitecture visualized by three-dimensional (3D) confocal microscopy," Histochemistry, vol. 100, no. 3, pp. 193-202, 1993.

[75] H. A. McNally and R. B. Borgens, "Three-dimensional imaging of living and dying neurons with atomic force microscopy," Journal of Neurocytology, vol. 33, no. 2, pp. 251-258, 2004.

[76] D. Comaniciu, P. Meer, and D. J. Foran, "Image-guided decision support system for pathology," Machine Vision and Applications, vol. 11, no. 4, pp. 213-224, 1998.

[77] D. Ristanović, N. T. Milošević, I. B. Stefanović, D. Marić, and I. Popov, "Cell image area as a tool for neuronal classification," Journal of Neuroscience Methods, vol. 182, no. 2, pp. 272-278, 2009. 


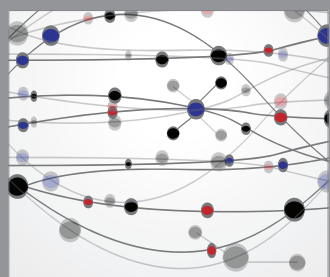

The Scientific World Journal
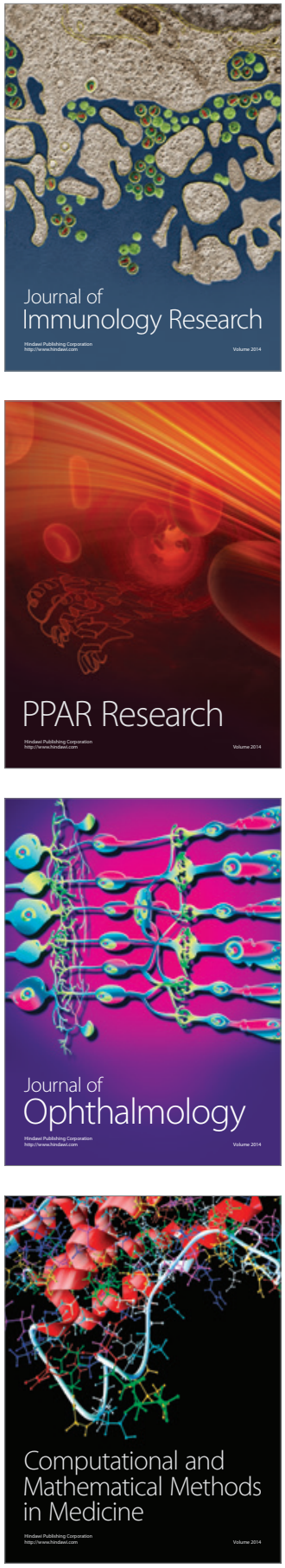

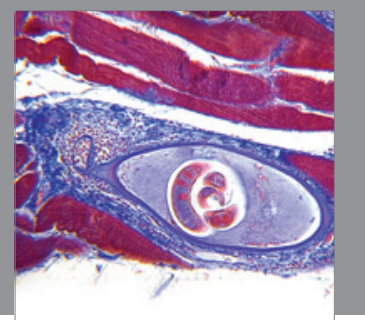

Gastroenterology

Research and Practice
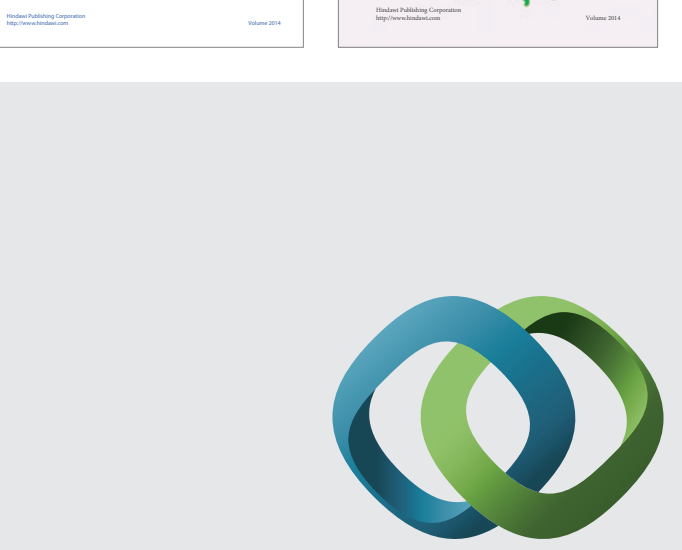

\section{Hindawi}

Submit your manuscripts at

http://www.hindawi.com
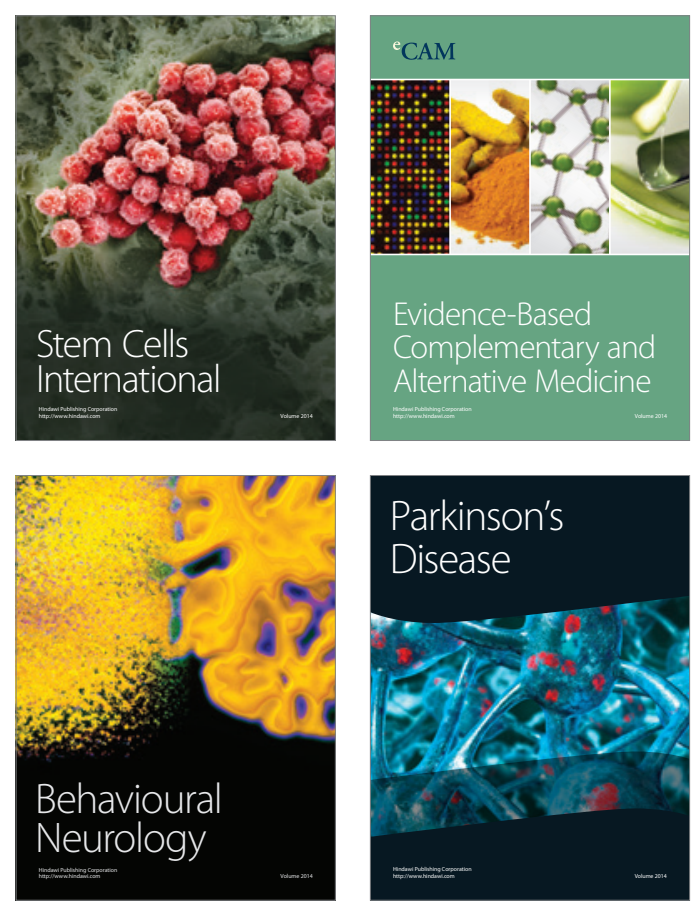

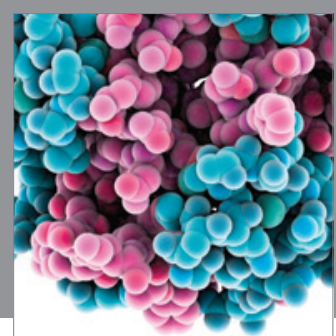

Journal of
Diabetes Research

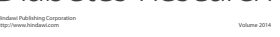

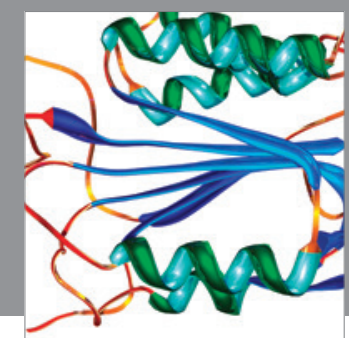

Disease Markers
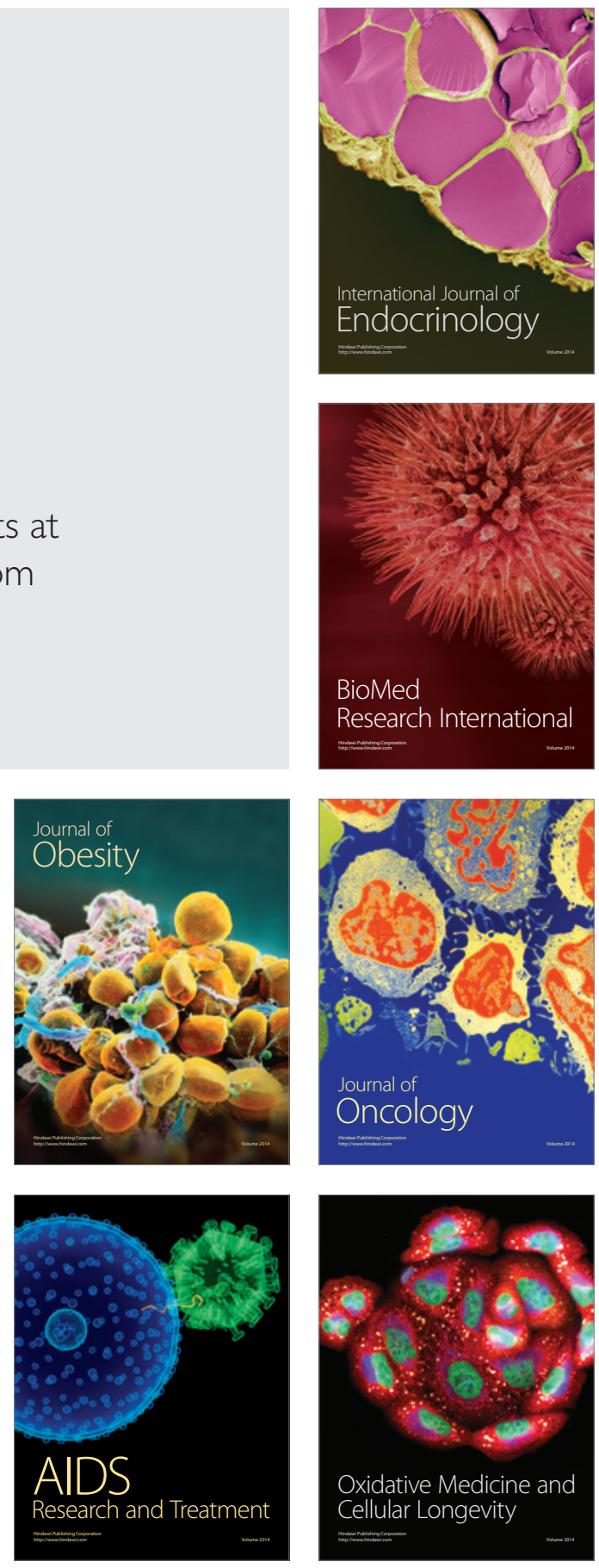